\title{
Pablo Marshall (ed.), Citizenship and Disadvantaged Groups in Chile
}

(2018) Lexington Books, Lanham [etc.], $221 \mathrm{pp}$.

\author{
Luis Lloredo Alix \\ Universidad Autónoma de Madrid \\ ORCID ID: 0000-0002-5483-7346 \\ luis.1loredo@uam.es
}

Cita recomendada:

Lloredo Alix, L. (2019). Pablo Marshall (ed.), Citizenship and Disadvantaged Groups in Chile. Eunomía. Revista en Cultura de la Legalidad, 17, 418-426.

doi: https://doi.org/10.20318/eunomia.2019.5048

El género de las recensiones es complejo y comprometido, y más aún cuando se trata de libros colectivos como el que en esta ocasión me propongo comentar. Me parece que podría optarse por tres estrategias diferentes. La primera consistiría en reseñar ordenadamente los títulos y los contenidos que integran la obra, con una perspectiva preferentemente neutral y descriptiva. La segunda consistiría en realizar una síntesis de conjunto, si es que el libro lo permite, para desde ahí tratar de encontrar hilos conductores o ejes argumentales susceptibles de presentar de forma crítica. La tercera, en fin, trataría de aunar las dos perspectivas anteriores, con el doble propósito de invitar a la lectura de los potenciales interesados -indicándoles qué pueden esperar del volumen y cuáles son sus contenidos principales- y al mismo tiempo de realizar un somero análisis crítico, señalando problemas de conjunto, proponiendo rutas argumentales alternativas o advirtiendo sobre fenómenos no abordados o inadecuadamente abordados. Modestamente, esta última es la perspectiva que trataré de adoptar en las páginas siguientes. Empezaré realizando algunas consideraciones sobre la obra en su totalidad, para después examinar los capítulos del libro en particular.

Lo primero es lo primero: Citizenship and disadvantaged groups es un buen libro. Como todos los libros colectivos, resulta inevitablemente desigual, porque los autores adoptan perspectivas metodológicas, estilos narrativos y marcos conceptuales diferentes, y porque no todos logran el mismo grado de precisión o agudeza en sus planteamientos. Sin embargo, creo que el grado de homogeneidad 
de los artículos y el nivel de exhaustividad de las problemáticas que se analizan es alto, superando con mucho a la mayoría de obras de este tipo, que a menudo degeneran lamentablemente en un cajón de sastre, como si fueran almacenes de artículos sin ligazón y sin vocación de conjunto. Esto es algo que ocurre frecuentemente con los libros de actas de congresos o con las obras colectivas que, no siendo compilaciones de ponencias en sentido estricto, son el resultado de seminarios de naturaleza heterogénea.

En esta ocasión, sin embargo, se trata de un escollo que el editor del volumen, el profesor Pablo Marshall, ha sabido evitar con acierto. Y lo ha logrado, en mi opinión, gracias al hecho de contar con un buen equipo de colaboradores, con ideas relativamente afines y una sensibilidad más o menos compartida respecto a los problemas de exclusión y desigualdad que caracterizan al Chile contemporáneo. Además, me parece que todos ellos comparten un punto de vista crítico respecto a las dos derivas que Pablo Marshall identifica como características del actual orden constitucional chileno -no solo en el sentido de Ley Fundamental, sino también en el de constitución histórico-social-, a saber: el neoliberalismo y el autoritarismo. El neoliberalismo y el autoritarismo son, en efecto, dos herencias de la dictadura pinochetista que han dado pie a un modelo económico y político profundamente desigual; a un modelo que, bajo la apariencia de ser democrático y liberal, se ha convertido en un generador de desigualdades difícil de enderezar (Cociña, Frei, Larrañaga y Rucks 2017).

Las dificultades de enmendar dicho orden obedecen a dos razones. Por un lado, la constitución creada por el jurista de Pinochet, Jaime Guzmán -fundador a su vez de un partido ultraconservador, la Unión Demócrata Independiente, que hoy en día sigue concurriendo a las elecciones con notable éxito- diseñó un entramado jurídico arduo de modificar, lleno de «cerrojos constitucionales». Esto ha llevado a un conocido filósofo del derecho chileno, Fernando Atria, a hablar de la «Constitución tramposa» (Atria, 2013). Por otro lado, dicha institucionalidad ha calado en el sistema político en sentido amplio y en la propia mentalidad de los chilenos, infiltrándose en la cultura y en las formas de conciencia social, y generando una dinámica que ni siquiera se ha podido subvertir en los periodos de gobierno de «izquierdas», al alero de la así denominada «Concertación». Así lo expresa el editor del volumen en su introducción:

As a result of the existing constitutional constraints and the consensual political culture of the Concertación, the almost three decades of civilian rule since 1990 have witnessed very slow progress in fields related to political democratization and social equalization and saw practically no significant distributive policies beyond the neoliberal logic of targeting social spending to the poorest members of society (2013, p. 4).

Estas dos características del orden constitucional chileno han terminado configurando una noción de ciudadanía excluyente, es decir, una ciudadanía asequible solo a las pocas élites culturales y a las oligarquías económicas -casi siempre coincidentes- que ya fueron beneficiadas durante los años de la dictadura militar y que, aún hoy, siguen copando las esferas de poder político y económico, con un exiguo ascenso social de las clases medias y una débil presencia institucional de las mismas. Esa ciudadanía excluyente, en efecto, se ha forjado de espaldas a las mujeres, a la clase trabajadora, al pueblo mapuche -y a los demás pueblos originarios que conviven dentro de las fronteras del Estado-, a las expresiones sexuales divergentes y, cómo no, de espaldas a la inmigración. Obviamente, la noción de ciudadanía siempre ha funcionado como un expediente de exclusión: al ciudadano le corresponden derechos, el ciudadano es concebido como sujeto político activo, el ciudadano cuenta en las estadísticas y en los programas de gobierno, el ciudadano 
tiene voz y es socialmente reconocido como participante de la experiencia colectiva; en cambio, el no ciudadano se ve condenado a la invisibilidad o la indiferencia, que a su vez suelen dar paso a la exclusión social, el expolio y la violencia. Con todo, hay ciudadanías más o menos inclusivas, más o menos porosas a incorporar sujetos y colectivos subalternos, más receptivas o más reacias a integrar otras formas de vida. Y la actual concepción de la ciudadanía chilena, como muestran los diferentes artículos que integran este volumen, se encuentra entre las más excluyentes.

Esto último es algo que merece conocerse más allá de las fronteras chilenas. Porque Chile ha logrado, gracias a una colosal operación propagandístico-cultural auspiciada por la ideología neoliberal triunfante y, en buena medida, por Estados Unidos- generar una imagen exterior que a veces resulta más halagüeña de lo que realmente es. Daré unos ejemplos a vuelapluma: al contrario de lo que sucede con muchos pueblos originarios de Latinoamérica, como el quechua, el aymara o el guaraní, que han alcanzado una notable visibilidad internacional, y cuya situación de opresión resulta bastante conocida, se sabe poco del enorme conflicto que existe en Chile en relación con el pueblo mapuche. Pongamos otro ejemplo: al contrario de lo que tendemos a opinar desde Europa, Chile ostenta el segundo puesto en violencia de género y el tercero en acoso sexual en América Latina (Yáñez, 2018). Por no mencionar el hecho de que, hasta hace apenas dos años, tenía una de las legislaciones sobre aborto más restrictivas del mundo, al prohibir la interrupción del embarazo en todas sus formas y casos posibles. Daré un último ejemplo: desde el extranjero, la imagen de Chile es la de un país en el que los niveles de pobreza son relativamente tolerables, en el que no existen esas brechas de desigualdad que caracterizan a muchos países de Latinoamérica. Sin embargo, si echamos un vistazo a los índices del Plan de Naciones Unidas para el Desarrollo, comprobaremos que Chile tiene un índice de desigualdad extraordinariamente alto (Jahan, 2018): actualmente se sitúa en el puesto número 44 del Índice de Desarrollo Humano, pero su índice de Gini le coloca como el país más desigual de la OCDE (Vergara Mattei, 2017). Además, si efectuamos la comparación con base en el coeficiente de desigualdad humana del PNUD -que no sólo tiene en cuenta la desigualdad de ingresos-, los resultados son llamativos: Chile alcanza un 14,9, superando a países como Singapur (11,9), Estados Unidos $(13,1)$, la República de Corea $(14,0)$, Grecia $(13,1)$, Argentina $(13,9)$ o Sri Lanka $(13,6)$. De los cincuenta países que encabezan el ranking del IDH, sólo Chile y España (también con un 14,9) llegan a una cifra tan alta en sus coeficientes de desigualdad.

Todo lo anterior hace que la aparición de este libro sea más que oportuna, sobre todo si tenemos en cuenta que se publica en inglés y con el evidente propósito de acceder a un auditorio amplio. Ahora bien, no pensemos que este talante divulgativo se limita al hecho del idioma, sino que atañe también al tono y el estilo de los artículos que integran el volumen. Con mayor o menor éxito, pero en casi todos los casos de forma palpable, los autores exponen sus respectivas problemáticas de una manera pedagógica, procurando situar al lector en los antecedentes sociopolíticos de cada cuestión y desgranando con cuidado los ingredientes legales, culturales e históricos de las diferentes situaciones de desigualdad que son objeto de su análisis. Esto hace que, junto al hecho de publicarse en inglés, la obra constituya una introducción difícilmente mejorable para quien quiera entender desde fuera las complejidades de la actual ciudadanía chilena. También en esto se siente el buen hacer del coordinador, Pablo Marshall, que a buen seguro se habrá ocupado de trasladar las correspondientes directrices estilísticas y metodológicas.

Dicho todo esto, y antes de entrar a resumir el contenido de cada capítulo, me gustaría lanzar dos breves apuntes críticos respecto a la obra en su conjunto. Ambos tienen que ver con ausencias que me parece detectar en el marco epistemológico de 
la obra. Se trata de dos vectores que, habida cuenta de los asuntos que se tratan y del modo que los autores tienen de abordarlos, podrían haberse incorporado de forma explícita. Si hacemos caso del contenido del libro y de la cantidad de capítulos que se dedican a analizar la situación de vulnerabilidad y desigualdad en que se encuentran las mujeres chilenas por diferentes causas -prostitución, violencia machista, prohibición del aborto, participación política, etcétera-, me parece que el enfoque feminista podría haberse subrayado como uno de los pilares del volumen. Lo mismo ocurre con el planteamiento decolonial: buena parte de los ensayos que encontramos en esta obra abordan problemáticas relacionadas con el tema del multiculturalismo y con el hecho de que, en buena medida, la desigualdad de muchos grupos sociales en Chile se debe a una política de explotación colonial. Del mismo modo que señalaba antes en relación con el feminismo, creo que podría haberse explicitado este factor como una de las orientaciones epistemológicas generales. Además, me parece que quizá habría sido deseable dividir el libro en bloques, tomando en consideración que varios de los asuntos tratados afectan a ejes transversales de la desigualdad. Obviamente, como ya podrán imaginarse, no se trata de críticas graves, sino de meras apostillas a una obra que, en su conjunto, me parece muy recomendable.

Ha llegado el momento de exponer someramente el contenido del libro, con vistas a orientar a los posibles lectores. El primer capítulo, a cargo de Fernando Muñoz, aborda varios de los problemas que hoy en día afectan a la comunidad de gays, lesbianas y transexuales en Chile. Bajo el título de «The Institutional Response to Sexually Diverse Citizenship. Readaptation and Resistance», el profesor Muñoz pasa revista a las transformaciones institucionales que se han producido en Chile en las dos últimas décadas, con el ánimo de abolir las trabas y discriminaciones legales que obstaculizaban -y aún lo hacen-el disfrute de derechos fundamentales por parte de las personas que se alejan de la hetero-cisnormatividad hegemónica. Partiendo de la constatación de que Chile fue el último país de Latinoamérica en abolir la prohibición de mantener relaciones sexuales entre personas del mismo sexo, Muñoz desarrolla un análisis «material» de la ciudadanía sexualmente diversa, es decir, no se contenta con levantar acta de las proclamas legales abstractas, sino que toma en consideración la posición social de los grupos afectados y los concretos arreglos institucionales que se han establecido para dar protección efectiva a tales grupos:

the degree of citizenship afforded to a particular collective demand is a function of the intensity of the political recognition gained by its bearers, which often is in its turn a function of the collective power of that particular group. It is in this sense that the state apparatus can be described, as Poulantzas suggested (1980), as an institutional condensation of relations of power and the complex and contradictory struggles to subvert them.

Desde estas premisas metodológicas, el capítulo examina varios episodios de lucha por el reconocimiento que han ido sentando las bases de la ciudadanía sexualmente diversa en Chile: los debates sobre la maternidad de las lesbianas, sobre la identidad de género y sobre la discriminación por orientación sexual.

A continuación, se suceden cuatro artículos con perspectiva feminista, que abordan varios problemas relacionados con la discriminación hacia las mujeres. El segundo capítulo, a cargo de la profesora Lieta Vivaldi, traza una breve reseña de la lucha por el derecho al aborto en Chile: «Autonomy, Vulnerability, and Resistance: Brief History of the Feminist Fight for Abortion». Se trata de una contribución especialmente importante para la historia reciente, dado que, hasta septiembre de 2017, Chile era uno de los pocos países del mundo en los que el aborto estaba prohibido bajo cualquier circunstancia. Además del interés que pueda tener conocer los avatares de esta problemática fuera de las fronteras chilenas, el capítulo es interesante por dos razones. Primero, porque afronta esta historia y sus diferentes 
episodios desde una perspectiva epistemológica foucaultiana, que le otorga un interés filosófico y sociopolítico que va más allá de la mera crónica de acontecimientos. Segundo, porque en la última parte del capítulo se analiza la cuestión del aborto farmacológico mediante Misoprostol, una técnica que ha cobrado una gran importancia en el activismo feminista chileno de los últimos años y que, desde el punto de vista de la autora, resulta especialmente relevante por las implicaciones biopolíticas que ha tenido su uso en un contexto prohibicionista como el chileno: por un lado, porque ha facilitado el control autónomo del cuerpo de las mujeres, aunque también, de forma ambivalente, su vulnerabilidad.

El tercer capítulo, de la profesora Yanira Zúñiga, desarrolla el tema de la participación política de las mujeres, bajo el título «Political Participation of Women. Potentialities, Limits, and Challenges». Seguimos en esta contribución con un enfoque epistemológico crítico y «material» de la desigualdad -del mismo modo que veíamos en el primero y en el segundo capítulo-, sólo que en este caso con mayor razón aún: por más que la participación política de las mujeres haya sido formalmente reconocida, siguen existiendo numerosos óbices sociales e institucionales para que dicha participación pueda ser considerada efectiva. El capítulo de Yanira Zúñiga va dirigido, precisamente, a hacer un análisis de estos obstáculos y a explorar las posibilidades de dos de los mecanismos correctores que se han ideado para hacer frente al desequilibrio entre hombres y mujeres a la hora de integrar los órganos de representación de nuestras sociedades: las cuotas y las medidas de paridad. La perspectiva institucional y parcialmente sociológica que emplea la autora es de agradecer, puesto que no se limita a una alabanza o una impugnación sin matices de este tipo de medidas, sino que da cuenta de sus dificultades de implementación en relación con los contextos socioculturales, y de cómo los arreglos institucionales concretos condicionan su grado de efectividad, más allá de las proclamas propagandísticas difundidas por los medios de comunicación. Al final del recorrido, veremos que el celo analítico de la autora nos proporciona guías firmes -aunque nunca definitivas, obviamente- para comprender con rigor y profundidad las diferencias entre dos clases de medidas anti-discriminatorias como las cuotas y la paridad. La conclusión de Zúñiga es la siguiente:

norms and discourses that promote a greater female presence in positions of power can mask forms of legitimization of the current order. Furthermore, the study of the leadership of Michelle Bachelet provides some elements that confirm the advantages associated with the politics of presence, but also raises questions about the true impact of new female leaders in the deconstruction of gender patterns.

El capítulo cuarto, a cargo de Paula Hollstein, se propone analizar las posibilidades y las dificultades de conceptualizar la violencia doméstica como un caso de tortura: «Torture and Domestic Violence. A View from Cases of Terroristic Torture and Intimate Terrorism». Debe tenerse en cuenta que la terminología de "violencia doméstica» es la que existe actualmente en la legislación chilena para caracterizar lo que, en otros contextos o desde otras ópticas políticas, denominaríamos violencia de género o violencia machista. Existen buenas razones para no hablar de violencia doméstica, sino de violencia machista, desde una perspectiva feminista. Sin embargo, sin negar lo anterior, la autora de este capítulo se propone tratar la violencia doméstica de forma separada a otras formas de violencia machista como la violación o la discriminación por género, para analizar con atención la plausibilidad -teórica y práctica- de concebir los casos de violencia doméstica como torturas desde un punto de vista jurídico. Se trata de una sugerencia que ya plantearon algunas feministas como Catherine MacKinnon, y que la profesora Hollstein explora en esta ocasión en el contexto del derecho chileno. El resultado es ciertamente interesante por las consecuencias político-jurídicas que acarrearía tal calificación -tengamos en cuenta 
que la violencia doméstica se suele abordar desde las categorías del derecho de familia y el derecho penal, mientras que la tortura es legalmente considerada como un crimen contra la humanidad-, así como por el enfoque conceptual y terminológico que nos brinda la autora: la noción de «intimate terrorism» que maneja resulta particularmente acertada y sugerente.

Llegamos así al quinto capítulo de la obra, con el que se pone fin al que podríamos denominar primer bloque del libro, en la medida en que todos los artículos mencionados hasta ahora analizan cuestiones relativas a la desigualdad por razón de género. En esta quinta contribución, de la mano de Paula Nuño y Ximena Valencia, se examina uno de los eternos caballos de batalla del feminismo: el trabajo sexual. Afortunadamente, al menos en mi opinión, la cuestión se plantea desde un enfoque parcialmente regulacionista, y teniendo claramente en mente la realidad política y sociocultural chilena: «The Response to Sex Work. A Regulation with a Voice of Their Own». Las autoras tratan de desmarcarse, en efecto, de un posicionamiento cerrado en las trincheras del regulacionismo y del abolicionismo, en el entendido de que ambas posturas suelen enarbolarse sin tener en cuenta las necesidades, los criterios y la voz de las propias afectadas. En el caso chileno, este enfoque es especialmente necesario, ya que existen dos grandes asociaciones de trabajadoras sexuales que se ocupan de reivindicar los derechos de las prostitutas y que, por ende, rechazan con fuerza el paternalismo de los planteamientos abolicionistas. Así pues, el propósito manifiesto de las autoras es «[to] explore the solution proposed by sex workers themselves to achieve full citizenship, namely the development of regulation that reflects their own voices».

El capítulo sexto de la obra podría considerarse, en cierto modo, como la bisagra entre dos secciones del libro, puesto que aborda el tema de la jurisdicción multicultural, analizando en particular el caso de las mujeres mapuche: «Towards a Multicultural Jurisdiction. The Case of Mapuche Women». Con este capítulo, el profesor Luis Villavicencio toca uno de los temas más sensibles para los Estados pluriculturales o plurinacionales, a saber, la apertura de la jurisdicción al pluralismo jurídico, estableciendo determinados espacios en los que la legalidad oficial del Estado cede en favor del respeto a determinadas prácticas consuetudinarias de los pueblos originarios. Luis Villavicencio comienza su artículo constatando la especialidad del caso chileno -el único país de América Latina que aún no ha reconocido constitucionalmente a sus pueblos indígenas- trazando una breve genealogía de las relaciones del pueblo mapuche con el Estado. A continuación, dedica un inevitable apartado a explorar el marco filosófico-político en el que cabe plantearse la legitimidad de las luchas por el reconocimiento de naciones, pueblos o culturas oprimidas durante la época colonial. Tanto la aproximación histórica como la teórica le llevan a concluir, en el siguiente epígrafe, la necesidad de superar «the narrow liberal citizenship ideal». Equipado con este aparato teórico, Villavicencio afronta, en las últimas secciones del artículo, el tema de la jurisdicción multicultural desde la perspectiva de la violencia contra las mujeres mapuche. Se trata de un tema espinoso, puesto que se trata de casos en los que, habitualmente, colisionan los imperativos del respeto a determinadas formas de gestión de los conflictos propias de los pueblos originarios, con la defensa de la dignidad y la integridad de las mujeres:

the challenge is to break through the binary solutions available and enable conciliation mechanisms that uphold the rights of women, as a minority within a minority. It is therefore imperative to advance toward a feminist discourse which, without denying the importance of culture, acknowledges women's subordinate status, stands for basic women's rights, and identifies the contextual circumstances that impact exclusion. 
La estrategia del autor para alcanzar este delicado equilibrio, que me parece acertada, pasa por integrar la perspectiva de la interseccionalidad y el enfoque deliberativo junto con el tema de la jurisdicción multicultural.

En el séptimo artículo del libro proseguimos con la senda iniciada por Luis Villavicencio. El profesor Matías Meza-Lopehandía aborda de nuevo las dificultades del multiculturalismo en un Estado, como el chileno, que está estructuralmente construido sobre un modelo económico neoliberal. Bajo el título «Frustrated Multiculturalism. (Neo) Liberalism and the Mapuche People», el autor examina el actual fenómeno de criminalización del pueblo mapuche y los obstáculos de desarrollar un programa coherentemente multicultural en el marco de un statu quo que se beneficia del expolio y la explotación del territorio mapuche. El autor parte de unas sucintas consideraciones históricas, para recalar a continuación en las tribulaciones políticas y sociales que ha arrojado la «nueva fe»: el giro multicultural. La conclusión a la que pretende arribar es que esa suerte de «multiculturalismo neoliberal», basado en el reconocimiento de ciertas diferencias culturales, pero incapaz de cuestionar el sistema de explotación del territorio mapuche ancestral, y reacio a conceder espacios de autonomía política real, ha entrado en crisis en las últimas décadas, desembocando en una fuerte operación de criminalización de los grupos o líderes que persisten en reivindicaciones «maximalistas». Asimismo, el autor pasa revista a las graves deficiencias en la implementación y aplicación de la consulta previa establecida en el Convenio 169 de la OIT, lo que constituye un fraude respecto a las expectativas que éste había generado en su momento. La conclusión del autor es, entonces, que la adopción del multiculturalismo en el marco del neoliberalismo ha conducido a comprender aquél como «a device for disciplining the Indian, mainly through resource allocation [...]. In any case, the days of multiculturalism seem to be over. The Mapuche movement has adopted the vocabulary of self-determination from years ago».

De estas dos últimas contribuciones sobre el multiculturalismo, enfocadas a reflexionar sobre la exclusión sufrida por el pueblo mapuche, transitamos hasta el capítulo octavo, que aborda un asunto que guarda algunas semejanzas con los anteriores, en tanto que también pone sobre el tapete las dificultades de construir una noción de ciudadanía no asimilacionista y asequible a las diferencias culturales. En efecto, bajo el título «Immigrants. Legal Configuration of a Disadvantaged Group», Jaime Bassa y Fernanda Torres se dedican a desgranar los consabidos obstáculos de incluir a la población migrante dentro del demos del Estado, un asunto que podría calificarse como una de las tensiones constitutivas del armazón teórico y práctico de los derechos humanos. Creo que el enfoque que adoptan los autores es el adecuado: a su modo de ver, el discurso de los derechos humanos tiene que ver con la idea de vulnerabilidad, de manera que los inmigrantes deben ser tratados, conceptualizados y diferenciados con base en los grados de vulnerabilidad que presentan. Este punto de vista les ha llevado a desarrollar un trabajo que estimo altamente recomendable, puesto que incorpora, en su primera parte, un análisis sociológico de las dinámicas migratorias en el Chile contemporáneo; desde el retablo ciertamente complejo y heterogéneo que queda dibujado tras este examen, los autores están en condiciones de hacer una crítica de la actual regulación de las migraciones, así como de proponer criterios de justicia -empíricamente fundados-orientados a diseñar un nuevo marco de derechos humanos de la población inmigrante en Chile. El punto de vista que manejan es fuertemente crítico respecto al estado actual de las políticas migratorias $y$, además, coincide con varios de los ensayos ya citados en lo que se refiere al enfoque institucional: más allá de las proclamaciones abstractas de las leyes, Bassa y Torres concluyen que la discrecionalidad administrativa que éstas confieren "can allow for discrimination and institutional racism, given the practices which that system allows». 
Pasamos, a continuación, al capítulo noveno del libro, en el que se examina un asunto de particular importancia para Chile, habida cuenta de que, como Pablo Marshall indicaba desde las coordenadas conceptuales del volumen, el autoritarismo es uno de los rasgos que caracterizan la historia y la política contemporánea de este país, incluso en el periodo post-dictatorial. Se trata, en efecto, de un ensayo titulado «Public Order and Inequality in the Streets», a cargo de Paz Irrarazábal, con el que se inicia la que podría considerarse como una tercera parte de la obra, dado que a partir de aquí se analizan varios problemas derivados de la aplicación del derecho por parte de las autoridades, y de las discriminaciones derivadas del uso de la fuerza estatal. De acuerdo con Irrarazábal, los casos de abuso policial, de uso indebido de la fuerza, discriminación en el ejercicio de la autoridad, etcétera, no se deben a «simply police malfeasance problems or individual wrongs», sino a arreglos institucionales concretos y a desigualdades estructurales que, después, se reflejan en la actuación policial. El análisis se apoya, además -como ya hemos visto en muchos otros artículos de este volumen- en un apreciable trabajo empírico derivado de varias entrevistas a ciudadanos, policías, activistas y agentes del Estado (desde carabineros a gendarmes, pasando por fiscales y otros). Ello hace que la discusión teórica que se desarrolla a continuación sea plenamente coherente y enfocada a un contexto real y que, por tanto, los frutos cosechados sean de indudable utilidad para suministrar criterios de justicia operativos. El análisis de casos que precede a las conclusiones doctrinales se dirige, como en casos anteriores, a varios colectivos vulnerables $u$ oprimidos que suelen ser objeto del acoso o la violencia policial. Una de las conclusiones a las que se arriban es que no existen apenas mecanismos de rendición de cuentas por parte de la policía, algo que se deriva de las carencias institucionales al respecto, pero también del hecho de que los objetivos de la vigilancia policial son casi siempre grupos vulnerables, que casi nunca elevan quejas o reclamaciones, pues se encuentran desincentivados por su situación de desamparo estructural y por la desconfianza radical hacia el Estado.

Si de ciudadanía se trata, uno de los temas centrales es el de la población reclusa. En efecto, junto al tema de las personas migrantes, el estatus de los presos y el acceso de éstos a los derechos siempre ha sido uno de los puntos de fuga de la teoría y la praxis de los derechos humanos. Por ello, el décimo capítulo esboza algunas reflexiones a este propósito en el marco del Chile contemporáneo. De la mano del coordinador del volumen, Pablo Marshall, y de la profesora Carla Moscoso, en este capítulo - «Citizenship and Prisoners»- se aborda un asunto que es particularmente agudo en Chile. En efecto, la privación de derechos políticos a las personas que han sido condenadas a penas superiores a tres años, algo que se establece nada más y nada menos que en la Carta Magna, plantea cuestiones de enorme magnitud para la teoría de los derechos. Parece, como los autores enuncian al principio de su artículo, que la Constitución chilena estableciese un nexo directo entre ciudadanía y crimen, lo cual ilustra respecto a la fragilidad de la concepción general de los derechos que existe en la cosmovisión del país. Frente a esta previsión constitucional, los autores elevan una serie de críticas que van desde un rechazo general a muchas prácticas del actual sistema penitenciario chileno, hasta una reflexión de mayor calado respecto a los requisitos que deben o no deben exigirse a la ciudadanía para ser titular de derechos fundamentales. Todo el texto está articulado por las ideas de inclusión y exclusión, que se manejan desde un punto de vista filosófico-político fecundo, no sólo para pensar acerca de la exclusión de la población reclusa, sino para reflexionar sobre los problemas intrínsecos de toda noción de ciudadanía y sobre la injusticia estructural que subyace a la construcción de toda comunidad política.

El último capítulo de la obra prosigue la senda de los dos ensayos anteriores -a saber, el cuestionamiento de las prácticas autoritarias del sistema jurídico-político- 
, pero en esta ocasión focalizándose en otro de los colectivos que tradicionalmente han sido objeto de reflexión cuando hablamos de desigualdad: el de los trabajadores. Con el título de "The Worker by Himself. Thwarting Workers' Collective Action Through Law», el profesor José Luis Ugarte expone las restricciones que se derivan del actual modelo laboral chileno en materia de derecho de huelga y de negociación colectiva. El eje argumental pasa por subrayar la esencial continuidad entre la dictadura y la democracia en lo que se refiere a las relaciones laborales. Pese a las reformas introducidas desde la llegada de la democracia, en efecto, la situación de subordinación de los trabajadores permanece prácticamente inalterada, ya que dichas reformas han sido meramente "periféricas» y no han atacado la causa principal de esta sumisión: la falta de poder colectivo de los trabajadores. Así las cosas, tras un análisis que se detiene en los aspectos históricos y jurídicos de esta situación -lo cual incluye consideraciones técnicas acerca del contrato individual de trabajo, pero también apreciaciones sociopolíticas acerca del exiguo papel de la sindicación-, el autor termina apostando por un fortalecimiento de los procedimientos colectivos de defensa de derechos laborales, en particular la negociación colectiva. El artículo es interesante porque, más allá de lo clásico del planteamiento anterior, Ugarte explicita las razones éticas y políticas que hacen de la negociación colectiva el mecanismo idóneo para alcanzar un ideal de igualdad medianamente coherente.

Hemos llegado así al final del libro, que se cierra con un índice de temas y nombres, algo que resulta de agradecer y que no abunda en la tradición bibliográfica latina. Como creo que puede desprenderse de la apresurada síntesis que he realizado en las páginas anteriores, la valoración general de la obra es positiva. Además de las consideraciones expuestas en las primeras páginas de esta recensión, habrá podido verse que existe una cierta afinidad de enfoques ideológicos y epistemológicos entre los autores de los respectivos artículos, lo cual hace que, pese a las inevitables disparidades, haya una coherencia difícil de encontrar en obras de esta naturaleza. Asimismo, creo que ha quedado patente que, en líneas generales, el libro adopta un estilo claro y divulgativo que ofrece una visión de conjunto idónea para lectores no chilenos. Por todo ello, no me queda sino volver a recomendar vivamente su lectura. Creo que estamos, en efecto, ante una saludable novedad editorial.

\section{Bibliografía}

Atria, F. (2013). La Constitución tramposa. Santiago de Chile, Chile: LOM ediciones. Cociña, M., Frei, R., Larrañaga, O., Rucks, S. et al. (2017). Desiguales. Orígenes, cambios y desafíos de la brecha social en Chile. Santiago de Chile, Chile: Programa de las Naciones Unidas para el Desarrollo.

Jahan, S. et al. (2018). Índices e indicadores de desarrollo humano. Actualización estadística de 2018. Nueva York, Estados Unidos: Programa de Naciones Unidas para el Desarrollo.

Vergara Mattei, C. (28 agosto, 2017). Chile, líder mundial en desigualdad y desprotección al empleo y los trabajadores. Nodal. Noticias de América Latina y el Caribe. Recuperado de https://www.nodal.am/2017/08/chile-lider-mundialdesigualdad-desproteccion-al-empleo-los-trabajadores-cecilia-vergara-mattei/ (fecha de consulta: 10 de septiembre de 2019).

Yáñez, C. (8 marzo, 2018). Chilenas entre las que más violencia y acoso sufren en el continente. La Tercera.

Recuperado

de https://www.latercera.com/tendencias/noticia/chilenas-las-mujeres-masviolencia-acoso-sufren-continente/91248/ (fecha de consulta: 10 de septiembre 2019). 